\title{
ADUBAÇÃO NITROGENADA NO CRESCIMENTO E PRODUÇÃO DE GLADÍOLOS EM LATOSSOLO VERMELHO DO CERRADO
}

\author{
Rebeca de Andrade Porto ${ }^{1}$; Márcio Koetz; Edna Maria Bonfim-Silva² ${ }^{2}$ Alessana Franciele \\ Schlitching ${ }^{3}$; Analy Castilho Polizel ${ }^{4}$; Raphael Pereira França de Paula ${ }^{5}$. \\ ${ }^{1}$ Mestranda em Engenharia Agrícola da Universidade Federal do Mato Grosso (UFMT), Campus Universitário de \\ Rondonópolis, Rondonópolis, Mato Grosso, Brasil, demornay@ hotmail.com \\ ${ }^{2}$ Professor Doutor da UFMT, Campus Universitário de Rondonópolis, Rondonópolis, Mato Grosso, Brasil, \\ marciokoetz@yahoo.com.br \\ ${ }^{3}$ Professora Doutora da UFMT, Campus Universitário de Rondonópolis, Rondonópolis, Mato Grosso, Brasil, \\ embonfim@hotmail.com \\ ${ }^{4}$ Mestranda em Engenharia Agrícola da UFMT, Campus Universitário de Rondonópolis, Rondonópolis, Mato Grosso, \\ Brasil, alessanamt@gmail.com \\ ${ }^{5}$ Professora Doutora da UFMT, Campus Universitário de Rondonópolis, Rondonópolis, Mato Grosso, Brasil, \\ analy@ufmt.br \\ ${ }^{6}$ Graduando do curso de Engenharia Agrícola e Ambiental da UFMT, Campus Universitário de Rondonópolis, \\ Rondonópolis, Mato Grosso, Brasil, raphael_franca_@hotmail.com
}

\begin{abstract}
RESUMO: Objetivou-se pelo presente estudo avaliar a adubação nitrogenada nas características produtivas do gladíolo, espécie Gladiolus $x$ grandiflorus L., variedade White friendship. O experimento foi realizado em casa de vegetação no período de julho a setembro de 2011, no Instituto de Ciências Agrárias e Tecnológicas, da Universidade Federal de Rondonópolis-MT. As parcelas experimentais foram constituídas por vaso de $13 \mathrm{dm}^{-3}$ contendo dois bulbos, em delineamento experimental inteiramente casualizado. Os tratamentos consistiram de seis doses de nitrogênio de $0 ; 25 ; 50 ; 75 ; 100$ e $125 \mathrm{mg} \mathrm{dm}^{-3}$, com quatro repetições, utilizando-se como fonte a uréia. Avaliou-se a altura de plantas, diâmetro de caule, tamanho da haste floral e número de flores do gladíolo. Após realização das análises estatísticas concluiu-se que as doses de nitrogênio não influenciaram os caracteres produtivos avaliados do gladíolo.
\end{abstract}

PALAVRAS-CHAVE: Gladiolus $x$ grandiflorus L., nitrogênio, nutrição de plantas.

\section{NITROGEN FERTILIZATION ON GROWTH AND YIELD OF GLADIOLUS IN AN OXISOL FROM SAVANNAH}

\begin{abstract}
The objective of the present study was to evaluate the nitrogen fertilization on the yield characteristics of Gladiolus, species Gladiolus x grandiflorus L., variety White friendship. The experiment was conducted in a greenhouse in the period from July to September 2011 in the Institute of Agrarian Science and Technology of Federal University of Rondonópolis, MT. The experimental plots were consisted of vessels of $13 \mathrm{dm}^{-3}$ containing two bulbs in completely randomized design. Treatments consisted of six nitrogen rates of $0 ; 25 ; 50 ; 75 ; 100 ; 125 \mathrm{mg} \mathrm{dm}^{-3}$ and four repetition using urea as source. It was evaluated the plant height, stem diameter, flower stem length and number of flowers of gladiolus. After carrying out the statistical analysis it was concluded that the nitrogen did not affect the productive evaluated characters of gladiolus.
\end{abstract}

KEY-WORDS: Gladiolus $x$ grandiflorus L., nitrogen, plant nutrition.

\section{INTRODUÇÃO}

O gladíolo também conhecido como

Palma ou Palma-de-Santa-Rita é uma flor de corte muito comum dentre as plantas ornamentais, sendo uma planta bulbosa de clima tropical e subtropical, possui folhas 
lanceoladas, caule denominado de escapo e inflorescências do tipo espiga floral. O número, tamanho e cor das flores são variáveis de acordo com a espécie ou cultivar. Possui bulbo sólido denominado de cormo e suas raízes são fasciculadas, ocorrendo em grande número (SIMÕES, 2001). Possui portes eretos, chegando até a 1,5 $\mathrm{m}$ de altura e, dependendo da espécie, apresenta grande variação na forma e tamanho das flores (SOUZA, 1970).

O gladíolo é de fácil condução, baixo custo de implantação, rápido retorno financeiro e possui ciclo curto. Esses fatores permitem o seu cultivo em pequenas áreas, com possibilidade de produção comercial de bulbos para os mercados interno e externo. Além disso, tem elevada importância econômica já que é dentre as flores de corte cultivadas no Brasil, o terceiro produto em volume depois das rosas e crisântemos.

As plantas de gladíolo se desenvolvem bem em condições de temperatura amena a quente, boa disponibilidade de água durante todo o ciclo e solos com boa drenagem, férteis e ricos em matéria orgânica. A produtividade normal chega a 300.000 hastes com flores por hectare, considerando-se a formação de uma haste por bulbo plantado (OLIVEIRA, 2009).

A cultura se desenvolve em duas fases, ciclo vegetativo que compreende da germinação ao florescimento, e o ciclo reprodutivo após o florescimento até o amadurecimento completo dos bulbilhos, aproximadamente 150 a 200 dias após o plantio (DAP). As variedades cultivadas não apresentam grandes diferenças quanto ao porte, número de flores e tamanho de espigas, mas se diferenciam quanto ao ciclo de floração, sendo: ciclo curto 65 DAP, ciclo médio 75 DAP, ou ciclo longo 85 DAP. Para a reprodução da cultura, os bulbos escolhidos devem ter uniformidade em tamanho e em quebra de dormência, para permitir uma colheita uniforme de flores (BARBOSA; LOPES, 2000).

Embora as cultivares, dentro de cada grupo tenham ciclos de floração bem uniformes, temperaturas elevadas e dias longos tendem a tornar a produção mais precoce, enquanto o inverso, baixas temperaturas e dias curtos, tendem a retardar a mesma (BARBOSA; LOPES, 1994).

O gladíolo responde bem com nutrição balanceada para uma máxima produção de flores e melhor desenvolvimento. A época de plantio e o manejo da adubação influenciam na produção e qualidade das flores de gladíolo, assim como nos bulbos e bulbilhos. Inadequada nutrição de planta causa desordens sérias e pode eventualmente levar para um declínio do vigor e produção da planta (HOSSIAN et al., 2011). Nitrogênio é um dos mais importantes nutrientes para respostas no crescimento e produção de gladíolos (LEGHRI et al., 2011). O nitrogênio no gladíolo é responsável pelo número das hastes florais e o número de botões florais produzidos por haste (GANCEBO, 2006). Sidhu e Arora (1989) observaram que o 
nitrogênio possui efeito significante na sanidade da flor em diferentes cultivares.

No Estado de Mato Grosso o cultivo de plantas ornamentais ainda é pequeno e mais focado no plantio de flores tropicais. As flores de corte como o gladíolo geralmente são trazidas de São Paulo onde possui um forte centro de produção. Estudos devem ser incentivados como forma de diminuir a dependência de outros estados e fortalecer a cadeia produtiva da região principalmente $\mathrm{o}$ produtor da agricultura familiar já que a floricultura é uma alternativa de complementação da renda.

Devido à escassez de informação com relação ao cultivo de gladíolos no estado de Mato Grosso e a recomendação quanto à sua adubação nitrogenada para o desenvolvimento da cultura. Objetivou-se pelo presente estudo avaliar a adubação nitrogenada na produção de gladíolos.

\section{MATERIAL E MÉTODOS}

$\mathrm{O}$ experimento foi realizado no período de junho a setembro de 2011, em casa de vegetação no Instituto de Ciências Agrárias e Tecnológicas da Universidade Federal de Mato Grosso, Campus Universitário de Rondonópolis-MT, localizado à 284 metros de altitude, $16^{\circ} 45^{\prime} \mathrm{S}$ de latitude e $54^{\circ} 56^{\prime} \mathrm{W}$ de longitude. No interior da casa de vegetação foi instalado um termohigrômetro para medir diariamente a umidade e temperatura do ar.

O solo foi coletado em área de vegetação de Cerrado nativo de um LATOSSOLO
Vermelho de textura média na profundidade de 0-20 cm, passado em peneira de $2 \mathrm{~mm}$ de abertura. Sua análise apresentou as seguintes características químicas e granulométricas: $\mathrm{pH}\left(\mathrm{CaCl}_{2}\right)=4,1 ; \mathrm{P}=2,4 \mathrm{mg} \mathrm{dm}{ }^{-3} ; \mathrm{K}=28$ $\mathrm{mg} \mathrm{dm}{ }^{-3} ; \mathrm{Ca}+\mathrm{Mg}=0,5 \mathrm{cmol}_{\mathrm{c}} \mathrm{dm}^{-3} ; \mathrm{Ca}=0,3$ $\mathrm{cmol} \mathrm{dm}^{-3} ; \mathrm{Mg}=0,2 \mathrm{cmol} \mathrm{dm}^{-3} ; \mathrm{Al}=1,1$ $\mathrm{cmol} \mathrm{dm}^{-3} ; \mathrm{H}=4,2 \mathrm{cmol} \mathrm{dm}^{-3} ; \mathrm{M} . \mathrm{O}=22,7 \mathrm{~g}$ $\mathrm{dm}^{-3}$; areia $=549 \mathrm{~g} \mathrm{Kg}^{-1}$; silte $=84 \mathrm{~g} \mathrm{Kg}^{-1} \mathrm{e}$ argila $=367 \mathrm{~g} \mathrm{Kg}^{-1}$; soma de bases $0,6 \mathrm{cmol}$ $\mathrm{dm}^{-3} ; \mathrm{CTC}=5,9 \mathrm{cmolc} \mathrm{dm}^{-3} ; \mathrm{V}=9,8 \%$.

$\mathrm{O}$ delineamento experimental foi inteiramente casualizado com seis tratamentos correspondentes à doses de nitrogênio: $0 ; 25$; 50; 75; 100 e $125 \mathrm{mg} \mathrm{dm}^{-3}$ utilizando-se como fonte a uréia, com quatro repetições. Cada unidade experimental foi constituída por um vaso de $13 \mathrm{dm}^{-3}$. Os bulbos de gladíolos foram adquiridos com tamanho de perímetro 12-14 $\mathrm{cm}$. Dois bulbos em cada vaso foram plantados a uma profundidade de $10 \mathrm{~cm}$ (Figura 1).

A espécie escolhida para o experimento foi o Gladiolus $x$ grandiflorus L., cuja variedade foi a White friendship, sendo esta de ciclo curto equivalente a 60 - 65 dias de cultivo (SEVERINO, 2007). A irrigação em todas as unidades experimentais foi mantida próxima à capacidade de campo.

Foi realizada uma calagem para elevação da saturação por bases para 70\%. Após a correção do solo, realizou-se adubação fosfatada $\left(\mathrm{P}_{2} \mathrm{O}_{5}\right)$ em todas as parcelas experimentais com $200 \mathrm{mg} \mathrm{dm}^{-3}$ na forma de superfosfato simples. A adubação potássica 
$\left(\mathrm{K}_{2} \mathrm{O}\right)$ foi de $50 \mathrm{mg} \mathrm{dm}^{-3}$ na forma de cloreto de potássio e sua aplicação foi parcelada juntamente com a adubação nitrogenada. A adubação com micronutrientes foi de $0,5 \mathrm{mg}$ $\mathrm{dm}^{-3}$ de boro na forma de $\mathrm{H}_{3} \mathrm{BO}_{3}$ e $1 \mathrm{mg} \mathrm{dm}^{-3}$ de zinco na forma de $\mathrm{ZnCl}_{2}$. A adubação com nitrogênio e potássio foi parcelada em quatro vezes, sendo aos 12, 24, 47 e 56 dias após o plantio. Ao longo do ciclo da cultura as plantas foram tutoradas com bambus a fim de evitar o tombamento das mesmas (Figura 2).

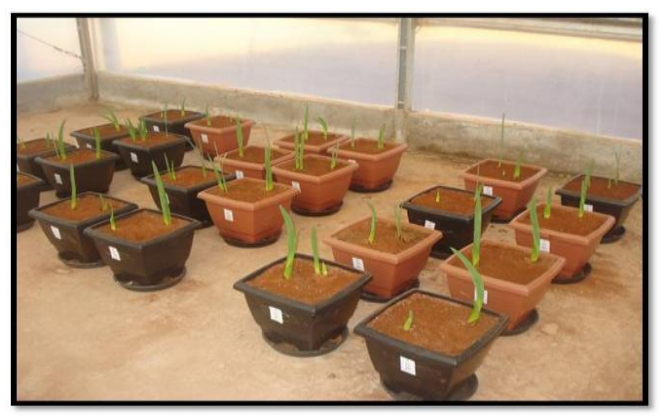

Figura 1. Vista do experimento aos 13 dias após o plantio.

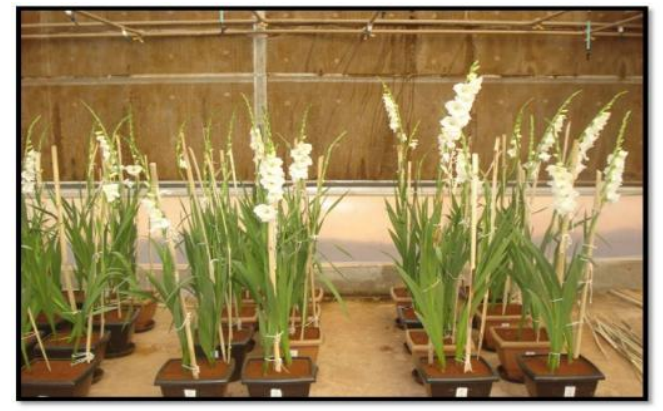

Figura 2. Gladíolos tutorados com bambu.
Os dados foram coletados aos 21 e aos 41 dias após o plantio (DAP) e quando $50 \%$ dos vasos estavam florindo. A caracterização do crescimento e produção do gladíolo foi avaliada pela medição do diâmetro de caule medido com o auxílio de uma paquímetro digital, a $5 \mathrm{~cm}$ de altura em relação ao solo (21 DAP); do número de folhas incluindo a folha bandeira (41DAP); da altura da planta medida com o auxílio de uma fita métrica do solo até a altura da folha mais alta ( 21 e 41 DAP); da altura da haste floral medida do solo até a altura final da haste floral e do número de flores. Estes dados foram coletados em todas as brotações que surgiram nos dois bulbos plantados em cada vaso, onde se realizou a média das medidas apuradas de todas as brotações de cada parcela.

Ao final do experimento as plantas foram classificadas de acordo com a graduação das hastes florais e número de flores segundo parâmetros de classificação da cooperativa de Holambra (Tabela 1). Os resultados foram submetidos à análise de variância, até $5 \%$ de probabilidade pelo teste F. Foi utilizado o software estatístico SISVAR para análise dos dados (FERREIRA 2000).

Tabela 1. Classificação brasileira de gladíolos para fins comerciais.

\begin{tabular}{lcc}
\hline Classe & Comprimento da haste $(\mathrm{cm})$ & Número de botões/flores \\
\hline Extra & $>120$ & $>16$ \\
I & $100-120$ & $12-16$ \\
II & de $80-120$ & $8-12$ \\
II & de $60-80$ & $<8$ \\
\hline
\end{tabular}

Fonte: Cooperativa agropecuária Holambra. 


\section{RESULTADOS E DISCUSSÃO}

As análises de variâncias dos dados de altura de plantas, diâmetro de caule, número de folhas e flores efetuada aos 21 dias, 41 dias e quando $50 \%$ das plantas estavam no florescimento mostraram que não houve efeito significativo em função das doses nitrogenadas estudadas. O número de gemas por bulbo que teve a dormência superada foi de 1,75 para cada bulbo acarretando assim, em uma colheita de quase duas flores por bulbo. Os gladíolos foram classificados na classe I, com média geral de haste floral de 1,41 m e número de flores com média geral de 13,47 sendo assim, dentro dos padrões comerciais (Tabela 2 e 3). Pode-se observar que a variedade White friendship se desenvolveu bem quando em condições climáticas de temperatura e umidade relativa média de $28,56{ }^{\circ} \mathrm{C}$ e $59 \%$ respectivamente (Figura 3 e 4).

Tabela 2. Síntese da ANOVA para as variáveis: número de folhas (NFO), diâmetro de caule (DC), altura de plantas aos 21 dias após o plantio (AP1), altura de plantas aos 41 dias após o plantio (AP2), comprimento da haste $(\mathrm{CH})$, número de flores (NFR).

\begin{tabular}{lccccccc}
\hline \multirow{2}{*}{ Fontes de Variação } & \multirow{2}{*}{ GL } & \multicolumn{5}{c}{ Quadrados Médios } \\
\cline { 3 - 7 } & & NFO & DC $(\mathrm{mm})$ & AP1 $(\mathrm{cm})$ & AP2 $(\mathrm{cm})$ & CH $(\mathrm{cm})$ & NFR \\
\hline Tratamentos & 5 & $0,476604^{\mathrm{NS}}$ & $6,831187^{\mathrm{NS}}$ & $43,701917^{\mathrm{NS}}$ & $36,881250^{\mathrm{NS}}$ & $0,016634^{\mathrm{NS}}$ & $3,294030^{\mathrm{NS}}$ \\
Erros & 18 & 0,794549 & 4,929778 & 16,044931 & 40,904375 & 0,007751 & 2,456328 \\
Total corrigido & & 23 & 23 & 23 & 23 & 23 & 23 \\
\hline Média & & 5,472 & 12,90 & 44,9666667 & 89,17 & 1,4170833 & 13,4775 \\
CV $(\%)$ & $16,29 \%$ & $17,20 \%$ & $8,91 \%$ & $7,18 \%$ & $6,21 \%$ & $11,63 \%$ \\
\hline
\end{tabular}

Onde: $\mathrm{CV}$ = coeficiente de variação; $\mathrm{NS}$ = não significativo.

Tabela 3. Médias observadas para as variáveis: número de folhas (NFO), diâmetro de caule (DC), altura de plantas aos 21 dias após o plantio (AP1), altura de plantas aos 41 dias após o plantio (AP2), comprimento da haste (CH), número de flores (NFR).

\begin{tabular}{ccccccc}
\hline \multirow{2}{*}{ Doses } & \multicolumn{7}{c}{ Médias observadas } \\
\cline { 2 - 7 } & NFO & DC $(\mathrm{mm})$ & AP1 $(\mathrm{cm})$ & AP2 $(\mathrm{cm})$ & CH $(\mathrm{cm})$ & NFR \\
\hline 0 & 5,375 & 12,355 & 48,350 & 90,9375 & 1,4475 & 12,8125 \\
25 & 5,750 & 15,305 & 40,2125 & 85,875 & 1,4475 & 14,4575 \\
50 & 5,075 & 11,895 & 48,0375 & 92,625 & 1,3775 & 12,8800 \\
75 & 5,875 & 13,190 & 44,600 & 90,8125 & 1,5125 & 14,5400 \\
100 & 5,675 & 12,640 & 42,050 & 85,000 & 1,3500 & 13,7675 \\
125 & 5,0875 & 11,975 & 46,550 & 89,500 & 1,3600 & 12,4075 \\
\hline
\end{tabular}

Lehri et al. (2011) observaram resposta positiva no comprimento das folhas, no número de folhas, no número de flores por haste, no comprimento das hastes com o aumento da adubação nitrogenada. Siraj e Al Safar (2006) tiveram resultados significativos com o aumento da adubação nitrogenada no comprimento de plantas, no número de folhas por planta, área da planta e seu peso seco.

De todos os nutrientes demandados pelo solo geralmente o nitrogênio é o que mostra efeitos mais significativos no crescimento e produção da planta. No entanto, alguns estudos sob o efeito da adubação nitrogenada 
em gladíolos têm reportado resultados contraditórios. Pandey et al. (2000) investigaram o efeito de diferentes níveis de nitrogênio no crescimento de gladíolos, não observando diferenças significativas para os parâmetros avaliados de peso da planta, comprimento das folhas, diâmetro da haste, dias para abertura da flor, comprimento das hastes e número de flores por haste.

Gancedo (2006) também não observou influência significativa de doses de nitrogênio nos valores médios de altura máxima de plantas, do comprimento da espiga e da haste floral. Hernãndez-Diaz et al. (2008) não observaram nenhuma diferença entre as doses de nitrogênio na qualidade das hastes. Butt (2005) estudando os efeitos da adubação com $\mathrm{N}, \mathrm{P}$ e K observou que estatisticamente todos os tratamentos em diferentes combinações de $\mathrm{N}, \mathrm{P}, \mathrm{K}$, não foram significativos para $\mathrm{o}$ comprimento das hastes e o que o tratamento com menor haste foi o de sem adubação e que esse último, não se diferenciou estatisticamente do tratamento somente com a adubação nitrogenada.

O tamanho do bulbo pode influenciar na resposta de adubação e o bulbo utilizado no presente experimento foi de tamanho 12-14 $\mathrm{cm}$ de perímetro e $4 \mathrm{~cm}$ de diâmetro. Há uma variação de incremento da produção de flores, qualidade da flor e a produção de bulbos e bulbilhos com o tamanho apropriado de bulbo (HOSSIAN et al., 2011). O crescimento inicial da planta e vigor são determinados pela quantidade de alimento fornecido para o crescimento da planta pelos bulbos (PANT, 2005). Memon et al. (2009) observaram que a altura da planta, largura da folha, comprimento da espiga de floração e número de flores por espiga foram significativamente influenciados pelo tamanho do bulbo.

A cultivar e o tamanho dos bulbos e bulbilhos também influenciam no requerimento de adubação do gladíolo (LEHRI et al., 2011). Bulbos menores requerem mais fertilizantes do que os bulbos maiores, principalmente devido à sua reserva armazenada e, em parte, à maior capacidade de alimentação do sistema radicular produzido por bulbos maiores (PAL, 2000). Além disso, segundo Tamura e Mega (1959) a produção de flores de bulbo de tamanho médio não é afetada por aplicações de fertilizantes.

Fernandes et al., (1974) avaliando três doses de N, P, K em culturas de gladíolos provenientes de três tamanhos diferentes de bulbos observaram que de modo geral as maiores respostas à adubação nitrogenada na produção de flores, bulbos e bulbilhos foram obtidas pela cultura proveniente do bulbo de menor tamanho (3 $\mathrm{cm}$ de diâmetro), e pelo bulbo jumbo (6 $\mathrm{cm}$ de diâmetro), enquanto que a cultura com o bulbo médio de $4 \mathrm{~cm}$ de diâmetro não houve resposta à adubação nitrogenada para o número de hastes florais. Fernandes et al., (1974) explicam ainda que isso pode ser devido haver uma maior exigência de nutrientes por parte de bulbos menores, principalmente devido à sua menor 
reserva nutricional. $\mathrm{O}$ bulbo de $4 \mathrm{~cm}$ de diâmetro não respondeu à adubação devido à sua reserva de nutrientes aliada à capacidade de um sistema radicular mais extensivo. No caso de bulbos jumbos seria de se esperar uma menor resposta do bulbo à adubação, contudo, isto não ocorreu devido à sua maior quantidade de reserva e por já estar em final de sua vida útil e menor vitalidade. Mesmo não havendo resposta do bulbo de $4 \mathrm{~cm}$ à fertilização, os valores de peso de bulbos, número de bulbilhos, comprimento de haste e número de botões florais por haste foram mais altos que os dos outros tamanhos de bulbos, indicando ser o mesmo possuidor de um maior vigor.

No experimento realizado presume-se que os bulbos cultivados e que apresentavam diâmetro de $4 \mathrm{~cm}$, possuíam reservas suficientes para o desenvolvimento da planta, não ocorrendo, portanto diferença significativa entre as doses de nitrogênio. Além disso, pode-se perceber o vigor desses bulbos já que os gladíolos se desenvolveram bem.

Há autores que ainda sugerem que respostas à adubação em plantios de bulbos de primeira safra não são observadas como foi o caso do presente experimento. Woltz (1955) sugeriu que a resposta do gladíolo com o aumento da adubação é relativamente lento quando comparado ao tomate e pode não ser aparente no primeiro plantio dos bulbos. Littrell e Waters (1967) afirmam que o incremento da produção de bulbos e flores com o aumento da adubação nitrogenada não foi observado até o segundo plantio dos bulbos.

Outro ponto a ser ressaltado é que o solo onde foi conduzido o experimento provavelmente não era deficiente em nitrogênio, já que não foi observado diferença entre o tratamento com as doses de nitrogênio quando comparado ao tratamento sem adubação. Esses resultados podem revelar que o solo possuía níveis de nitrogênio suficientes para o desenvolvimento da planta. Solo e clima de uma determinada região são fatores importantes que influenciam a exigência de adubação do gladíolo (LEHRI et al., 2011). Os conteúdos de nutrientes encontrados nas plantas estão determinados por fatores bióticos e abióticos como temperatura, umidade relativa, luminosidade, genótipo, concentração de nutrientes no solo, cultivar e manejo do cultivo; por este motivo se pode encontrar valores diferentes de conteúdo de nutrientes, já que dependem das condições específicas de onde se desenvolveu as pesquisas (ABDALLA et al., 2002; JARVAN; POLDMA, 2004).

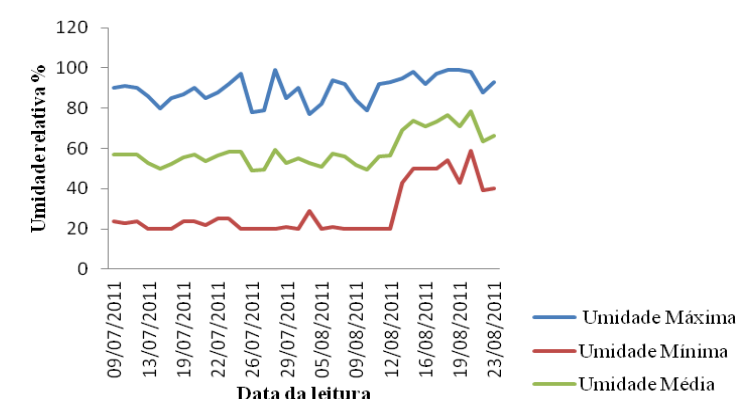

Figura 3. Umidade relativa máxima, mínima e média do ar dentro da casa de vegetação durante o período do experimento. 


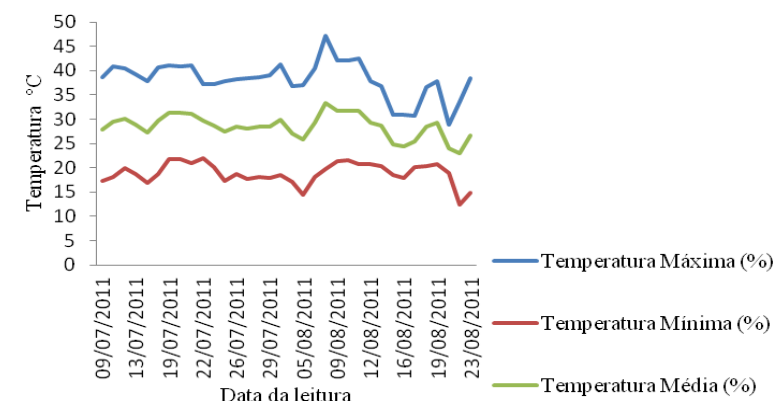

Figura 4. Temperatura máxima, mínima e média do ar dentro da casa de vegetação durante o período do experimento.

\section{CONCLUSÃO}

$\mathrm{O}$ aumento das doses de nitrogênio não proporcionou alteração no crescimento e produção comercial do gladíolo.

\section{REFERÊNCIAS}

ABDALA, J.; REZANDE, P.; AMERICO, A.; FINGER, F. L.; FERREIRA, F. A. Absorção de nutrientes pelo tomateiro cultivado sob condições de campo e de ambiente protegido. Horticultura Brasileira, v. 20, p. 90-94, 2002.

BARbosA, J. G. Palma de Santa Rita (gladíolo): produção comercial de flores e bulbos. Viçosa: Imprensa universitária, 2011. $113 \mathrm{p}$.

BARBOSA, J. G.; LOPES, L. C. O cultivo de gladíolo. Viçosa: Imprensa Universitária, 1994. $13 \mathrm{p}$.

BUTT, S. J. Effect of N, P, K on Some Flower Quality and Corm Yield Characteristics of Gladiolus. Tekirdağ Ziraat Fakültesi Dergisi Journal of
Tekirdag Agricultural Faculty. Tekirdag Turkey. p. 212-214. 2005.

FERREIRA, D. F. Manual do sistema Sisvar para análises estatísticas. Lavras, Universidade Federal de Lavras, 2000. 66p

GANCEBO, M. Efeito do nitrogênio, calcário e gesso agrícola em alguns atributos de um Latossolo e no desenvolvimento de gladíolo. 2006. 59f. Dissertação (Mestrado em Agronomia)Universidade Federal do Mato grosso do Sul. Dourados-MS.

HERNÃNDEZ-DIAZ， M. I.; MARREROGONZALEZ, V.; GONZALEZ-HURTADO, M. Nitrogen levels and their fractioning in gladiolus cultivation for Ferralitic Red soils. Pesquisa Agropecuária Brasileira, v.43, n. 1, p. 21-27, 2008.

HOSSIAN, M. J.; AMIN, M. R.; UDDAIN, J. Effect of corm size and fifferent doses of phosphorus on the grouwth, corm and cormel development of gladiolus. Lybian agriculture research center journal international, v. 2, n. 1, p. 9-14, 2011.

JARVAN, M.; POLDMA, P. Content of plant nutrients in vegetables depending on various lime materials used for neutralising bog peat. Agronomy Research, v.2, p.39-48, 2004.

LEHRI, S. M.; KURD, A. A.; RIND, M. A.; BANGULZA, N. A. The response of Gladiolus tristis L. to N and P2O5 fertilizers. 
Sarhad Journal of Agriculture, v. 27, n. 2, 2011.

LITTRELL, R. H.; WATERS, W. E. influence of nitrogen and lime fertilization on gladiolus corm and flower production and internal microflora of corms. Florida Agricultural Experiment Stations Journal Series, n. 2852, 1967.

OLIVEIRA, L. L. Produção de gladíolo em função da aplicação de nitrogênio e ethiltrinezapac, 2009. 43 f. Dissertação (Mestrado em Agronomia)- Universidade Estadual Paulista Júlio de Mesquita, campus Ilha solteira, Ilha Solteira- SP, 2009.

MEMON, Noor-un-Nisa; QASIM, M.; JASKANI, M. J.; AHMAD, R.; ANWAR, R.

Effect of various corm sizes on the vegetative, floral and corm yield attributes of gladiolus. Pakistan Journal of Agricultural Sciences, v. 46, n. 1, 2009.

PAL, P. R. Effect of nitrogen, phosphorus and plant density on growth and yield of flower in (Jasminum auriculatum L.) Var. Single. South India Hort., v. 32, n. 3, p. 140-146, 2000.

PANDEY, R,K.; PUNEET, R.; SINGH,M.K.; RATHORE, P. Effect of different levels of nitrogen and phosphorus on gladiolus under Agra conditions. Journal of Ornamental Horticulture, v. 3, p. 6-61, 2000.
PANT, S.S. Effect of different doses of nitrogen and phosphorus on the corm and cormel development of gladiolus (Gladiolus sp.) cv. american beauty. Journal of the Institute of Agriculture and Animal Science. v. 26, p. 153-157, 2005.

SEVERINO, C. A. de M. Cultivo comercial de Palma de Santa Rita Gladiolus sp. Tourm. Rede de Tecnologia da Bahia RETEC/BA, maio de 2007, dossiê técnico. Serviço Brasileiro de Respostas Técnicas SBRT. Disponível em: <http://www.sbrt.ibict.br>.

SIDHU, G. S.; ARORA, J. S. Response of gladiolus varieties to nitrogen application. Indian Journal of Horticulture, v. 46, n. 2, p. 250-54, 1989.

SIMÕES, F. C. Propagação in vitro de gladíolo (Gladiolus x grandiflorus L.). 2001. 65f. Dissertação (Mestrado em Fitotecnia) Universidade Federal de Lavras, Lavras, 2001.

SIRAJ, Y. S.; AL-SAFAR, M. S. Effect of GA3 Treatment and Nitrogen on Growth and Development of Gladiolus Corms. Pakistan Journal of Biological Sciences, v. 9; p. 25162519, 2006.

SOUZA, H. M. Cultura de gladíolos. São Paulo: Sociedade Brasileira de Floricultura. 1970. 12 p. 
TAMURA, J.; MEGA, K. Yelds of Gladiolus

Flowers and Corms Supplied with Differential Nutrients. Bulletin Hortic. Abstracts, Univ. Osaka, Pref. Ser. B, v. 9, n. 33, p. 7-41, 1959.
WOLTZ, S. S. Effect of differential supplies o nitrogen, potassium, and calcium on quality and yield of gladiolus flowers and corms. Proceedings of the American Society for Horticultural Science, v. 65, p. 427-435, 1955. 